\title{
Low carbon steel corrosion damage prediction in rural and urban
}

\section{environments}

\author{
V. Díaz", C. López* and S. Rivero*
}

\begin{abstract}
This paper presents an Artificial Neural Network (ANN) model for the damage function of carbon steel, expressed in $\mu \mathrm{m}$ of corrosion penetration as a function of environmental variables. Working in the context of the Iberoamerican Atmospheric Corrosion Map Project, the experimental data comes as result of the corrosion of low alloy steel subtracts in three test sites in Uruguay, South America. In addition, we included experimental values obtained from short time kinetics studies, corresponding to special series from one of the sites. The ANN numerical model shows attractive results regarding goodness of fit and residual distributions. It achieves a RMSE value of $0.5 \mu \mathrm{m}$ while a classical regression model lies in the range of $4.1 \mu \mathrm{m}$. Furthermore, a properly adjusted ANN model can be useful in the prediction of corrosion damage under different climatological and pollution conditions, while linear models cannot.
\end{abstract}

Keywords Atmospheric corrosion. Damage function. Neural networks. Pollution. Rural and urban environments.

\section{Predicción de la corrosión del acero de bajo carbono en intemperie rural y urbana}

\begin{abstract}
Resumen Este artículo presenta la metodología de las redes neuronales artificiales (RNA) como solución para el modelado de los valores experimentales obtenidos en los procesos de corrosión atmosférica. Se desarrolla el modelo de RNA para la función de daño, expresada en $\mu \mathrm{m}$ de penetración para el acero de bajo carbono en función de las variables medioambientales, en el contexto del Proyecto MICAT (Mapa Iberoamericano de Corrosión Atmosférica) y programas de experimentación propios. Los datos experimentales son resultado de los estudios de calibración sobre sustratos ferrosos en tres sitios del territorio uruguayo, Sudamérica. Se incluyen, además, los valores experimentales obtenidos en los estudios de cinéticas iniciales, correspondientes a series especiales de cortos tiempos de exposición en una de las estaciones de ensayo. El modelo numérico de RNA muestra resultados con un valor de RMSE de $0,5 \mu \mathrm{m}$, en tanto el modelo de regresión clásico arroja un valor de $4,1 \mu \mathrm{m}$.
\end{abstract}

Palabras clave Corrosión atmosférica. Función de daño. Redes neuronales. Contaminación. Ambientes rural y urbano.

\section{INTRODUCTION}

Uruguay takes part of the collaborative project MICAT $^{[1]}$, operating four atmospheric corrosiveness stations. Standardized metallic samples are exposed in different natural environments, using also standardized procedures and environmental data collection agreed by the MICAT working group. Amongst meteorological ones we include hourly values of relative humidity, temperature, wind speed and direction, as well as daily precipitation and precipitation run in number of days. Pollution data includes monthly values of sulfur dioxide and chloride deposition rates.

There is a significant research body regarding analytical expressions or models ${ }^{[2-6]}$. The economical and technological importance is fairly clear, because meteorological and even pollution records are taken routinely, while the corrosion rate of low alloy steel require mid-to-long term experiments. The task has shown to be difficult, because of nonlinearity's associated with the physicochemical process responsible for the atmospheric corrosion phenomena.

$\left(^{*}\right)$ Facultad de Ingeniería-Universidad de la República Oriental del Uruguay, J. Herrera y Reissig 565, C.P. 11300, Montevideo. Uruguay. 
Most of the predictive models used to date are linear regression models that fit the data such that the root mean square error is minimized. Nevertheless, they have been shown to be effective only in few areas. Artificial Neural Network Modeling emerges as a promising tool in corrosion research, because of it potential to model complex non linear processes provided its architecture and parameters are properly set.

This paper tackles the modeling of corrosion penetration in terms of standard meteorological variables for low carbon steel alloy. We have selected as pertinent meteorological parameters the cumulated time of wetness (in h), cumulated chloride deposition (in $\mathrm{mg} \mathrm{Cl} / \mathrm{m}^{2}$ ), cumulated sulfate deposition (in $\mathrm{mg} \mathrm{SO} / \mathrm{m}^{2}$ ), cumulated hours with relative humidity below $40 \%$ (in h), cumulated precipitation (in $\mathrm{mm}$ ), as well as mean relative humidity (in \%), and mean temperature (in ${ }^{\circ} \mathrm{K}$ ).

The problem under investigation is one of function estimation. Given a set of $\mathrm{n}$ observations at time $i$ of $m$ meteorological variables $X_{i}$ and the corresponding observed corrosion penetration values $f_{i}, i=1 \ldots n$, find $f(\mathbf{X}, \mathbf{P})$ (boldface denotes a vector) such that the mean sum of the squared errors MSSE, defined as:

$$
\operatorname{MSSE}(p)=\frac{1}{n} \sum_{i=1}^{n}\left(f\left(X_{i}, P\right)-f_{i}\right)^{2}
$$

is minimized with respect to the function parameters $P$.

$f(X, P)$ is the function providing the corrosion penetration of carbon steel, while $X$ is a vector of cummulated meteorological variables (in our case, $\mathrm{TDH}, \mathrm{Cl}, \mathrm{SO}_{2}, \mathrm{P}, \% \mathrm{HR}$ less than $40 \%$ ).

\section{MATERIALS AND METHODS}

Low alloy steel subtracts, with known chemical composition (C $0.05 \%$, Mn $0.37 \%$, S $0.011 \%$, P $0.01 \%$, Cr $0.012 \%$, Ni $0.012 \%$, Cu $0.021 \%$, Ti $0.02 \%$, As $0.01 \%$ ), prepared according to ISO 8407 Standard $^{[7]}$, were exposed to atmospheric corrosiveness stations, covering very pure rural to industrial-urban polluted atmospheric environments. The experimental design comprised long and short term exposure series. Concerning long-term exposure periods, all the test sites have had the following exposure schedule: 3 series with one-year exposure, and one serie with two, three and four years exposure periods, respectively. Concerning shortterm, an experiment was designed including five sequences corresponding to: $1,2,3,5$ and 7 months of exposure periods for samples located at a continental industrial-urban test site named Site 2. Four specimens of each serie were exposed in each sequence, three of which were used to evaluate annual corrosion rate. The specimens were exposed in each test sites corresponding major climatological parameters described in table I.

\subsection{Brief introduction to artificial neural networks}

The term "artificial neural network" (ANN) denotes a computational structure intended to model the properties and behavior of the brain structures, particular self-adaptation, learning and parallel processing. It consists of a set of nodes and a set of interconnections between them ${ }^{[8 \text { and } 9]}$. A node contains a computational element (neuron) which takes inputs through synaptic connections from some other neurons and produces a single output. A typical neuron takes as input the weighted sum of the input links and produces a single output through a given transfer function. The behavior and properties of such a net is dependent of the computational elements, in particular the weights and the transfer function, as well as the net topology. Usually the net topology and the transfer function are specified in advance and are kept fixed, so only the weights of the synaptic connections, and the number of neurons in the hidden layer need to be estimated. The

Table I. Some climatological parameters for the measuring stations

Tabla I. Algunos de los parámetros climatológicos en las estaciones de medida

\begin{tabular}{lrrr}
\hline \multirow{3}{*}{ Parameters } & \multicolumn{3}{c}{ Stations } \\
\cline { 2 - 4 } & Site 1 & Site 2 & Site 3 \\
\hline $\mathrm{tm}^{(1)}\left({ }^{\circ} \mathrm{C}\right)$ & 10.5 & 10.9 & 11.1 \\
$\mathrm{tM}^{(2)}\left({ }^{\circ} \mathrm{C}\right)$ & 24.3 & 23.0 & 23.2 \\
$\mathrm{t}^{(3)}\left({ }^{\circ} \mathrm{C}\right)$ & 16.9 & 16.7 & 17.0 \\
$\mathrm{Pm}^{(4)}(\mathrm{mm} /$ month) & 85.8 & 81.1 & 79.3 \\
$\mathrm{PM}^{(5)}(\mathrm{mm} /$ month) & 121.6 & 115.3 & 121.1 \\
$\mathrm{R}^{(6)}(\mathrm{mm} /$ year $)$ & 1201.2 & 1088.9 & 1138.1 \\
\hline
\end{tabular}

1: mean coldest month temperature, 2: mean hottest month temperature, 3: mean annual temperature, 4: minimum monthly rain, 5: maximal monthly rain, 6: mean annual rain. All stations are located in areas classified as Humid Template Without Dry Season, according to Köppen scale. 
usual procedure is to divide the available dataset in two parts: the learning (or training) set and the test set. The former is presented to the ANN, and the Mean Sum of Squared Errors MSSE $(P)$ is minimized by properly choosing of $P$. This process is denoted as training. Once $P$ is estimated, a verification takes place analyzing the generalization ability of the ANN, evaluating the MSSE using the test set. Usually, this new value is larger than the one obtained for the training set; if they are similar or even smaller, the parameters are accepted and the ANN is ready to be used. Otherwise, some adjustment should be made, and the overall training process is repeated. Notice that the parameters are held constant while evaluating the MSSE with the test set.

The transfer functions can be linear or not. The latter ones are more often used because they allow the network to fit better the training set than linear ones. Linear ANN can only map linear functions, severely limiting the usefulness of the model for our purposes.

The neurons can be connected in many ways, and thus leading to different architectures. The most popular option is named as Multilayer Perceptron Structure. It consists of one input layer, where the connection with the input data is performed. Such input is passed (lets say, from left to right) to a hidden layer. All neurons in the first hidden layer receive a weighted average of all inputs, but there is no connection among them. If available, further hidden layers receive inputs from weighted averages of the outputs of the previous layers. The final layer collects the inputs of the last hidden one, and produced the output of the ANN. In our case, we have just one neuron in the final layer, because we will produce just a function value. Each hidden layer can have any number of neurons, and each neuron can have (in first instance) a different transfer function. The more neurons in the hidden layers, the more sophisticated cases the network is capable of learning.

All the neurons receive as input a weighted average of the outputs of the previous layer; we will denote the weights as $w_{i j}$. Each synaptic weight $w_{i j}$, is interpreted as the strength of the connection from the $j^{\text {th }}$ unit to the $i^{\text {th }}$ unit. It is customary to add a constant bias term is added in order to improve the training phase. The input can be calculated as

$$
\text { NetInput }_{j}=\mu_{j}+\sum_{i} w_{i j} * \text { output }_{i}
$$

where $w_{i j}$ are the synaptic connection weights from neuron $j$ to neuron $i$, output $t_{i}$ is the output from neuron $i$, and $\mu_{j}$ is a bias for neuron $j$.

The output of each neuron is a simple function of its net input. It can be linear, but the most interesting case is the non-linear one. A number of nonlinear functions have been used by researchers as transfer or activation functions, for example:

$$
g(\text { NetInput })=\left(1+e^{- \text {NetInput }}\right)^{-1}
$$

(Also denoted as the sigmoid function)

$$
\begin{aligned}
& g(\text { NetInput })=\sinh (\text { NetInput }) \\
& g(\text { NetInput })=\tanh (\text { NetInput }) \\
& g(\text { NetInput })=\operatorname{asinh}(\text { NetInput })
\end{aligned}
$$

Cybenko $^{[10]}$ demonstrated that under weak requirements on the transfer function, an ANN with one hidden layer and enough neurons can approximate any continuous function to an arbitrary degree. The synaptic weights in neural networks are conceptually similar to coefficients in regression models. They are adjusted to solve the problem presented to the network.

Learning or training is the term used here to describe the process of finding the most suitable values of these weights. There are many different algorithms for training neural networks. Backpropagation $^{[11]}$ is the most popular one. It modifies the weights by moving in the direction contrary to the MSSE error function gradient. The algorithm is limited by the fact that it is sensitive to the set of initial weights, and it may get trapped in local optima; it requires in addition that the transfer function should be differentiable.

\subsection{ANN modeling}

In order to facilitate the learning process, the input variables were normalized to a zero mean, unitary variance equivalents. We used in the following the same name for the input variable or its normalized version. The output of the ANN needs not to be normalized. In other branches of science, the $\Pi$ Buckingham Theorem plus the prior knowledge of all significant variables allow a reduction in the number of inputs to the ANN while keeping valid the resulting model. Up to now it is impossible to take full advantage of this, since the variables that control the corrosion process are in general not precisely known. 
The dataset is rather small, so it has been artificially enlarged. It is well known in the ANN literature that some noise in the input is beneficial in terms of convergence, so the learning set were duplicated using a random perturbation of at most $5 \%$. The final training set was composed of 51 observations, plus 51 more obtained by perturbation. The test set have 18 records.

The number of neurons for the input and output layer are fixed by the number of meteorological variables (five) and the number of variables to be predicted (one). The design of the ANN will be completed once the number of neurons in the hidden layer and the activation function is specified. Usually the activation function has considerable less influence in the final results ${ }^{[10]}$ so the asinh function has been selected for the hidden layer, and the linear one for the output neuron.

As a working tool it has been used the Matlab Neural Networks Toolbox 2.0, in the context of Matlab 4.2 c.

\section{RESULTS AND DISCUSSION}

This section presents an Artificial Neural Network model for the estimation of a damage function for low carbon steel substrates as a function of some input variables. Later we will discuss the performance of the model in terms of goodness of fit, and the residual distributions for training and testing data sets will be analyzed.

The inputs variables considered are: $X_{1}$ time of wetness (in h), $\chi_{2}$ sulfate deposition (in $\mathrm{mg}$ $\left.\mathrm{SO}_{2} / \mathrm{m}^{2}\right), X_{3}$ precipitation (in $\mathrm{mm}$ ), $X_{4}$ hours with relative humidity below $40 \%$ (in $\mathrm{h}$ ), $\chi_{5}$ chloride deposition (in $\mathrm{mg} \mathrm{Cl} / \mathrm{m}^{2}$ ), all of them cumulated over the considered period. The target value is the cumulated penetration (in $\mu \mathrm{m}$ ). We recall that during training, we normalized the input variables to zero mean, unit variance.

The full ANN model's output can be expressed as follows:

$y_{1}=\operatorname{asinh}\left(W_{1} X+B_{1}\right) ; \quad y_{2}=B_{2}+W_{2} \cdot y_{1}$

where $X$ is the vector of input variables normalized to a zero mean, unitary variance. The scalar output $y_{2}$ should fit the measured penetration (without normalization).

It should be pointed out that the model with two neurons in the hidden layer shown the best fitting capabilities, exhibiting the smallest RMSE both for learning and testing data sets. This will be presented below. The final weights for the ANN with two neurons in the hidden layer are:

$$
\begin{aligned}
& W_{1}=\left(\begin{array}{rrrrr}
-0.8346 & 0.8445 & -0.3661 & 0.2571 & -0.6190 \\
2.0439 & -27.1515 & 5.4670 & 3.3881 & 2.6605
\end{array}\right) ; \\
& B_{1}=\left(\begin{array}{r}
-0.3199 \\
3.0145
\end{array}\right)
\end{aligned}
$$

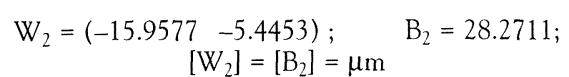

$$
\begin{aligned}
& \text { Standard deviation }=\left(\begin{array}{lllll}
6439 & 4977.4 & 1644.6 & 225.1 & 4779.6
\end{array}\right) \\
& \text { Required for the normalization } \\
& \text { Mean }=\left(\begin{array}{lllll}
7364.1 & 3422.5 & 1935.3 & 221.2 & 3171.3
\end{array}\right)
\end{aligned}
$$

With such parameters, the RMSE for the training set was $0.5 \mu \mathrm{m}$, while for the test set it was $2.5 \mu \mathrm{m}$ which has been considered acceptable. These results are graphically represented in the figure 1 , additionally with the distribution of the discrepancies between the ANN output and the measurements as well as the evolution of the penetration in function of the time of exposure which are respectively represented in figures 2 and 3 .

To make a comparison, we also used a classical linear regression model with the same normalized inputs. The weights were $\mathrm{W}=(-14.010417 .0326$ $29.0991-7.3892-4.2913)$ and the constant term was $26.4930 \mu \mathrm{m}$. In this case, the training set were
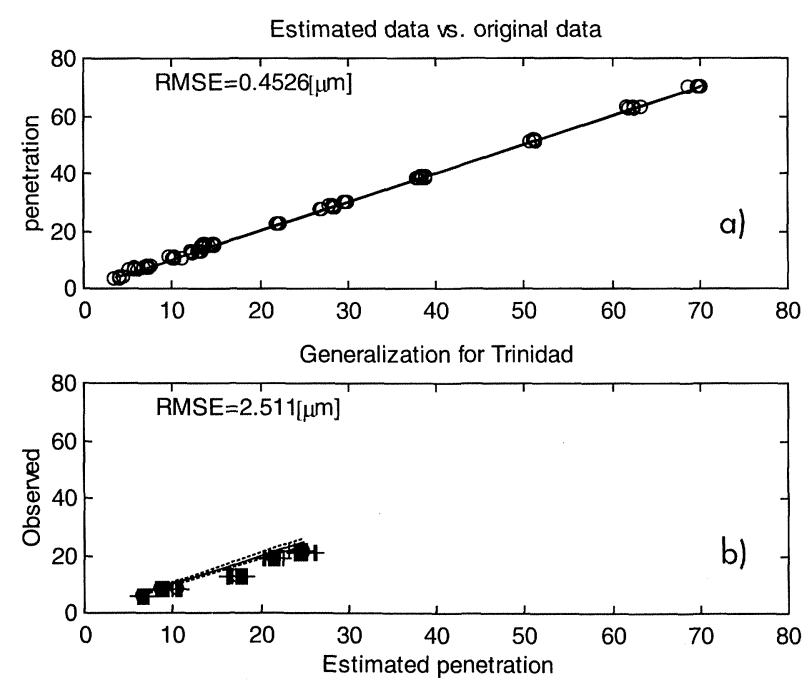

Figure 1. a) Comparison of measured vs. calculated penetration values (in $\mu \mathrm{m}$ ) for the training set. b) The same, for the testing set.

Figura 1. a) Comparación de los valores medidos contra los calculados (en $\mu \mathrm{m}$ ) para el conjunto de entrenamiento. b) Lo mismo, para el conjunto de prueba. 

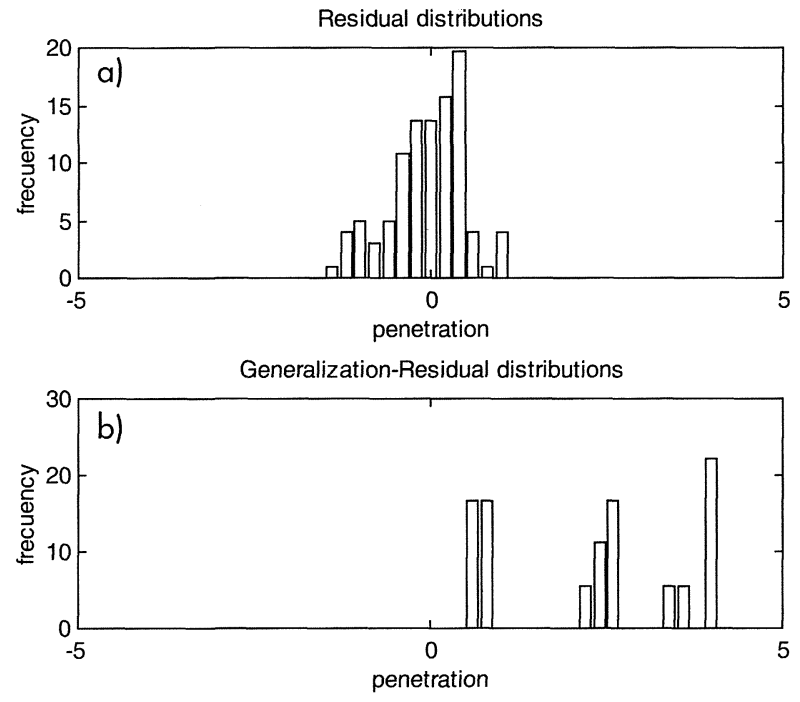

Figure 2. Distribution of the discrepancies between the ANN output and the measurements. a) For the training set, b) for the testing set.

Figura 2. Distribución de las discrepancias entre la salida ANN y las medidas. a) Para el conjunto de entrenamiento y b) para el conjunto de prueba.

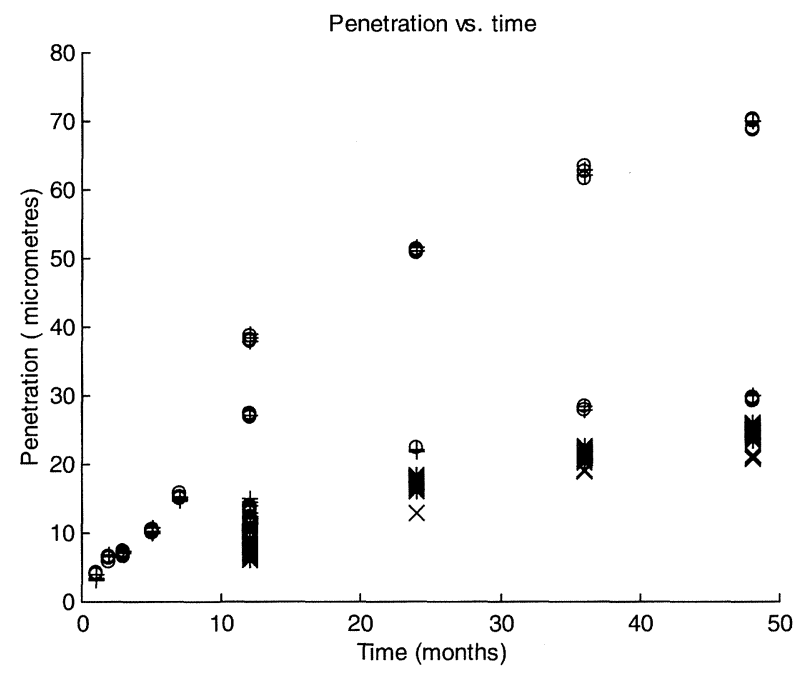

Figure 3. Evolution of the cumulated penetration vs. time of exposure. "o" estimated values and " + " observed values, both for training set. "*" estimated values and " $x$ " observed valuesm both for testing set.

Figura 3. Evolución de la penetración acumulada contra tiempo de exposición. "o" valores estimados $y$ " + " valores observados, ambos para el conjunto de entrenamiento. "*" valores estimados $y$ " $x$ " valores observados, ambos para el conjunto de prueba.

fitted with an RMSE of $4.1 \mu \mathrm{m}$, and the same figure for the test data set was $4.6 \mu \mathrm{m}$. This show that the generalization capabilities of the linear model were good. However, the comparison with other values in the literature should be made with caution, because the researchers usually made the fit with all the available data, and reports the RMSE with such set; in that case, the ANN produces $0.5 \mu \mathrm{m}$ while the linear model $3.9 \mu \mathrm{m}$.

\section{CONCLUSIONS}

- This paper presents an ANN-based methodology for the modeling of atmospheric corrosion functions, and the full model obtained with data from Uruguay. Its validity to other environments is yet to be ascertained.

- Meteorological variables as mean relative humidity (in \%), and mean temperature (in ${ }^{\circ} \mathrm{K}$ ) shown no relevance in modeling both training and testing data sets. Taking into account these meteorological variables the RMSE for the training set is $0.4 \mu \mathrm{m}$ while for the test set is $2.1 \mu \mathrm{m}$.

- Results were clearly improved by including the hours with $\mathrm{HR}<40 \%$. If this variable is not included the results are a RMSE of $0.6 \mu \mathrm{m}$ for the training set, and $5.6 \mu \mathrm{m}$ for the test set.

- It showed excellent results regarding goodness of fit and residual distributions on learning and testing data sets.

- Residual distributions corresponding to the test sites are not normal ones nor correspond to the same distribution.

- The ANN model holds promise to be useful in the prediction of corrosion damage under different climatological and pollution conditions and this solution methodology is expected to be an effective and efficient tool for the construction of models associated with atmospheric corrosion processes for other metals, and in general, in the modeling of corrosion processes from experimental data.

\section{REFERENCES}

[1] M. Morcillo, ASTM STP 1239, Kirk W.W, Lawson H.H., eds. American Society for Testing and Materials, Philadelphia, 1995, pp. 257-275.

[2] R.H. Mc Cuen and P. Albrecht, ASTM STP 1194 , American Society for Testing and Materials, Philadelphia, 1994, pp. 65-101.

[3] J.W. SPEnCE and F.H. HAYNIE, Corrosion 48 (1992) 1.009 1.019 .

[4] S. Feliu and M. Morcillo, Corros. Sci. 34 (1993) 415 422. 
[5] S.D. Cramer, B.S. Corvino and G.R. Holcomb, 13th International Corrosion Congress 1996, Melbourne, Australia, paper 032, 1-9.

[6] I. COLE, 13th International Corrosion Congress 1996, Melbourne, Australia, paper 035, 1-8.

[7] ISO/DIS 8407 Standard, Genève, Switzerland, 1985.
[8] G. Cybenko, Math. Control Signals Syst. 2 (1989) 303-314.

[9] D. E. Rumelhart and J.L Mc Clelland, Parallel Distributed Processing 1,2 MIT Press, Cambridge, MA, 1986.

[10] H.S. STERN, Technometrics 38 (1996) 205-220.

[11] B. Wagner and M. MisRa, Am. Stat. 50 (1996) 284-293. 